\title{
THE INTEGRATION OF PSYCHOLOGICAL AND NETWORK PERSPECTIVES IN ORGANIZATIONAL SCHOLARSHIP
}

\author{
Tiziana Casciaro, University of Toronto ${ }^{1}$ \\ Sigal Barsade, University of Pennsylvania \\ Amy Edmondson, Harvard University \\ Cristina Gibson, University of Western Australia \\ David Krackhardt, Carnegie-Mellon University ${ }^{2}$ \\ Giuseppe (Joe) Labianca, University of Kentucky
}

\begin{abstract}
Although multiple disciplines have been applied to the study of organizations, organizational research is rarely interdisciplinary in the sense of two or more disciplines being linked in the joint analysis of organizational phenomena. The articles in this special issue illustrate the kinds of insights that can be gained by moving from a purely disciplinary perspective on organizational behavior to an interdisciplinary perspective that considers network phenomena and psychological phenomena as intertwined in organizational life. The advances of this special forum notwithstanding, large swaths of network-psychology integration are still largely unexplored in organizational research. We highlight a subset of particularly promising avenues for further interdisciplinary exploration. We also observe that the two research programs have developed into distinct paradigms, making interdisciplinary discourse challenging, and we offer suggestions toward a greater integration and collaboration across the two research communities.
\end{abstract}

Our understanding of organizations comes from a wealth of differing theoretical domains and perspectives. Yet organizational research rarely integrates these different domains, despite repeated calls in the literature and arguments in support of the benefits of doing so (e.g., Gioia and Pitre, 1990).

Disciplines, and literatures within disciplines, instead tend to develop separate theories and approaches, with little cross-fertilization. This special issue attempts to cross-fertilize psychological, micro-level organizational research with a more sociologically-based, macro-level organizational domain - the network paradigm. Doing so is important not only for enhancing our specific understanding of workplace networks, but also for illustrating how an interdisciplinary approach can better illuminate the complexity of organizational life.

\footnotetext{
${ }^{1}$ All authors after the first are listed alphabetically and contributed equally.

${ }^{2}$ David Krackhardt's contribution to this work is supported in part by the Singapore National Research Foundation under its International Research Centre @ Singapore Funding Initiative and administered by the IDM Programme Office.
} 
We argue that network and psychological studies of organizations are complementary and can synergistically improve our understanding of organizational phenomena. In its most extreme form, the structuralist perspective underlying many network studies of organizations de-emphasizes (Wellman and Berkowitz, 1988) or denies altogether (Mayhew, 1980) the importance of social actors' individual characteristics and psychological processes in favor of explanations of organizational phenomena focusing on the patterns of relationships linking actors to one another, and the topological and positional configurations that drive actors' behavior. While a purely structuralist approach may usefully explains neural, molecular, and other networks in the physical world, organizational networks connect feeling, thinking human beings. As social networks, the structural patterns of relationships that emerge in organizations unavoidably implicate human psychology. Acknowledging this distinctively human trait, early developments in social network research linked seamlessly social structures and psychological processes (e.g., Bavelas, 1950; Heider, 1958; Homans, 1961; Leavitt, 1951; Moreno, 1961; Simmel, 1950). Over time, however, disciplinary boundaries hardened, making such cross-disciplinary advances less frequent in network research. Reversing this trend, research in management has been showing signs recently of a renewed interest in joining structural sociology and psychological approaches in the study of organizations, with social network scholars drawing on psychological processes and micro-organizational behavior scholars adopting network concepts and methods.

These advances notwithstanding, social network research has yet to fully leverage the contributions of psychological research to the understanding of organizational networks. Likewise, these more psychological approaches to organizational studies have not fully integrated the sophisticated view of social context provided by network research. When we study deeply the interplay of psychological and network phenomena as joint keys to understanding organizational phenomena, we open many possible new research questions, as represented by the multiple forms in which the three domains of inquirynetworks, psychology, and organizational phenomena — can be modeled in relation to one another, as Figure 1 and 2 illustrate. 
Figure 1. Disciplinary perspectives on organizational behavior
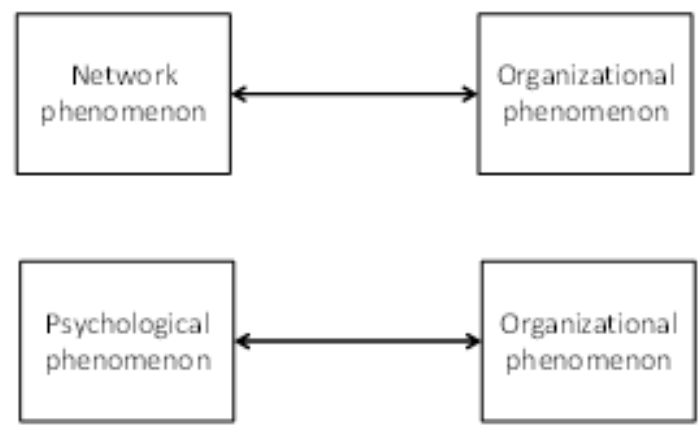

Figure 2. An interdisciplinary perspective on organizational behavior

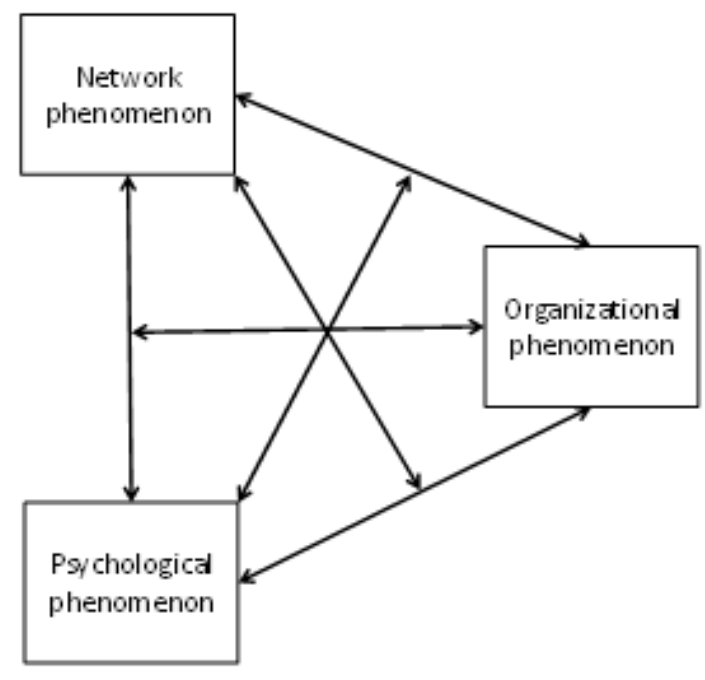

The articles in this special issue illustrate the kinds of insights that can be gained by moving from a purely disciplinary perspective on organizational behavior (Figure 1) to an interdisciplinary perspective that considers network phenomena and psychological phenomena as intertwined with organizational 
phenomena or outcomes (Figure 2). For example, James Vardaman, Shannon Taylor, David Allen, Maria Gondo, and John Amis (2015) begin with the observation that, although turnover intentions are the most common cognitive phenomenon explored as an antecedent to turnover, they explain only 15 to 20 percent of variance in actual turnover (Griffeth, Hom \& Gaertner, 2000). Combining this insight with recent research suggesting that employees are embedded in webs of relationships and attachments within organizations that create a "sense of stuckness" (Mitchell et al. 2001), Vardaman et al. (2015) proposed that network characteristics would moderate the relationship between turnover intentions and subsequent turnover, serving as enticements to stay rather than leave the organization. Investigating teachers in public schools and nurses in a hospital setting, they found that employees who intend to turnover were less likely to do so if they were central in advice and friendship networks (see Figure 2a). Practically speaking, this suggests that managers help to develop interpersonal relationships among employees to increase attachment and obligation which can subsequently reduce turnover. From a theoretical perspective, this study demonstrates that psychological and network factors jointly impact turnover, with network characteristics serving as an important boundary condition on previously uncovered psychological phenomenon.

Figure 2a. An interdisciplinary perspective on turnover (Vardaman, et al., 2015)

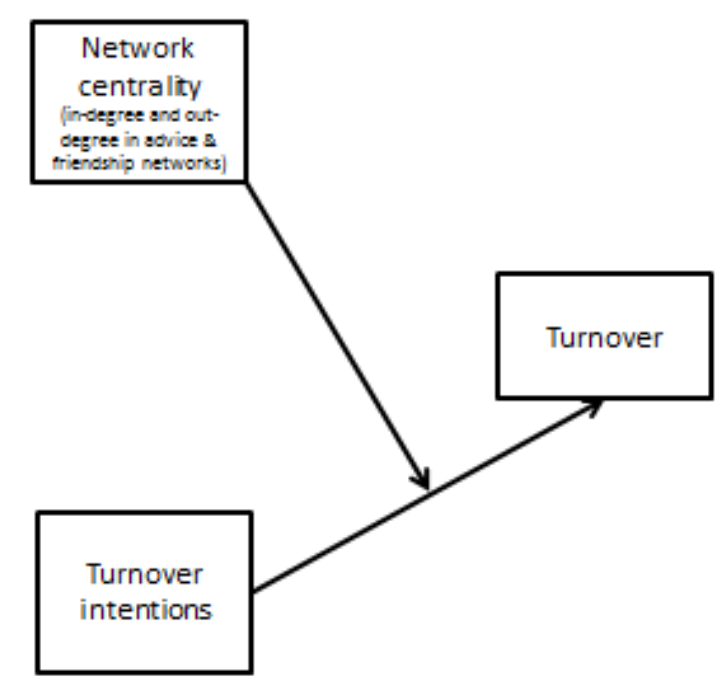


The research questions opened up for investigation by integrating networks and psychology are numerous, because such integration allows researchers to examine the joint effects of many organizational phenomena that network and psychological paradigms have investigated separately. To help define this vast domain of inquiry, we first identify three foundational levels of phenomena explored in network research — the topology of a network as a whole; the structural position of individuals in the network; and the dyadic ties or relationships in the network. We then identify four basic psychological phenomena that have often been the focus of psychological studies of organizations-personality/individual differences; cognition; affect; and motivation—as candidates for interdisciplinary research with network studies to develop more insightful models of organizational phenomena. We discuss how psychological and network perspectives may be integrated, including explaining how the authors in this special issue have begun to do so. Lastly, we discuss forms of interdisciplinary integration that are still largely unexplored, yet have great potential to advance organizational knowledge.

Table 1. Network and psychological phenomena as building blocks of interdisciplinary inquiry

\begin{tabular}{|c|c|}
\hline Network (structural) phenomena & Psychological phenomena \\
\hline $\begin{array}{l}\text { I. The network as a whole (topology) } \\
\text { II. The individuals in the network } \\
\text { (positions-roles) } \\
\text { III. The dyads in the network } \\
\text { (relationships) }\end{array}$ & $\begin{array}{l}\text { i. Affect } \\
\text { ii. Cognition } \\
\text { iii. Personality } \\
\text { iv. Motivation }\end{array}$ \\
\hline
\end{tabular}

\section{A network-psychological perspective on organizations}

\section{Network (structural) phenomena}

The study of social networks focuses attention on the pattern or structure of relations among a set of actors. For example, while traditional explanations of career success and leadership might focus on an employee's training or intelligence, a social network perspective emphasizes the importance of the employee's position and structural connections in the web of workplace relationships. While leadership is 
often conceived as a set of personal abilities and skills, a network perspective considers the relationships within which a leader is embedded - such as the relations between the leader and her followers, or the bridging role the leader provides to outside groups, or even the pattern of relations among her followers each of which might enhance or inhibit the leader's effectiveness.

Social network analysis can add to our understanding of social phenomena through investigations of different units of analysis and levels of analysis (Krackhardt, 2010). Unit of analysis refers to the aggregation of people into units of interest as primary actors in a system. Social network theories and methods are sufficiently multifaceted to apply to units as diverse as people (e.g., networks of friends), organizations (e.g., networks of joint ventures), industries (e.g., networks of transactions), or even nations (e.g., networks of trade). Given our special issue theme, we restrict ourselves to a discussion of the social networks where people are the unit of analysis.

Level of analysis, in contrast, is more complex as it does not refer to simple hierarchical aggregations of smaller units into larger ones. A network is seen as a system of interdependent units (people). Rather than assume independence or try to remove interdependence statistically, as often done in organizational studies, network theorists view the structure of interdependency as the primary focus of their research; it is the crucial lens for understanding organizational behavior. To understand how this distinctive perspective gives rise to types of theorizing distinct from non-network paradigms, we begin by identifying the three most common levels of analysis conducted in network research.

The Network as a Whole (Topology). The first level of analysis addresses such questions as: What is the overall shape of the network? How do we characterize that shape? And what effect does this shape have on the performance and behavior of the larger group as a whole? Different shapes have different implications for what people see, how they think, and how the group or system of players will behave. For example, a group in which everyone's interaction funnels into one central person is highly centralized and would act differently than a group with the same number of ties, but disbursed among two subgroups. Network measures also can be used to define a group’s centralization. In Figures 3a and 3b 
(borrowed from Krackhardt, 2010), both networks are about the same size, and they have approximately the same number of overall ties - but their shape is quite different. Figure 3a is a stylized core-periphery structure (Everett and Borgatti, 1999) with a small group of people (the core) with ties among each other and ties to those on the periphery. Those on the periphery have ties to the core but relatively few ties to each other. Such a structure represents another type of highly centralized work group, one that might be efficient for executing tasks that are relatively simple and routine (Shaw, 1964). However, this structure can also become hierarchical, because the core acts in ways to reinforce its power advantages and the periphery becomes disenchanted with the resulting inequity. This may lead to negative group dynamics that undercut the efficiencies of this structure.

\section{Figure 3a - Core-Periphery Structure}

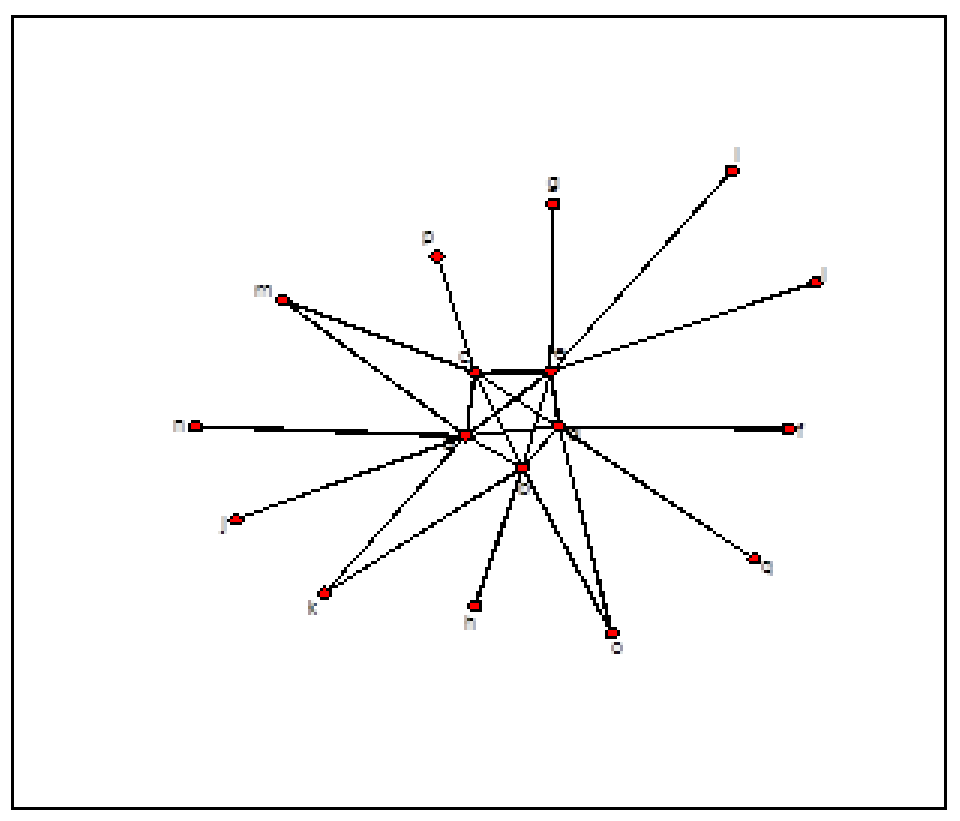

The structure in Figure 3b represents a very different shape, a classic bow-tie that shows two relatively densely connected subgroups connected by one bridging individual. The integrity of the overall structure is fragile, because it is dependent on one person $(\mathrm{O})$, without whom the subgroups would be separated into two non-interacting components. Not only is the overall structure fragile, the coherence within subgroups, combined with the lack of connection between subgroups, tends to devolve into a local group identity, a “we-them” attitude, as Sherif and colleagues (1961) found in the famous Robbers Cave 
experiments. If the group's purpose is to perform an overall integrated task, then this structure might interfere with necessary cross-group cooperation and coordination.

Figure 3b - Bow Tie Structure

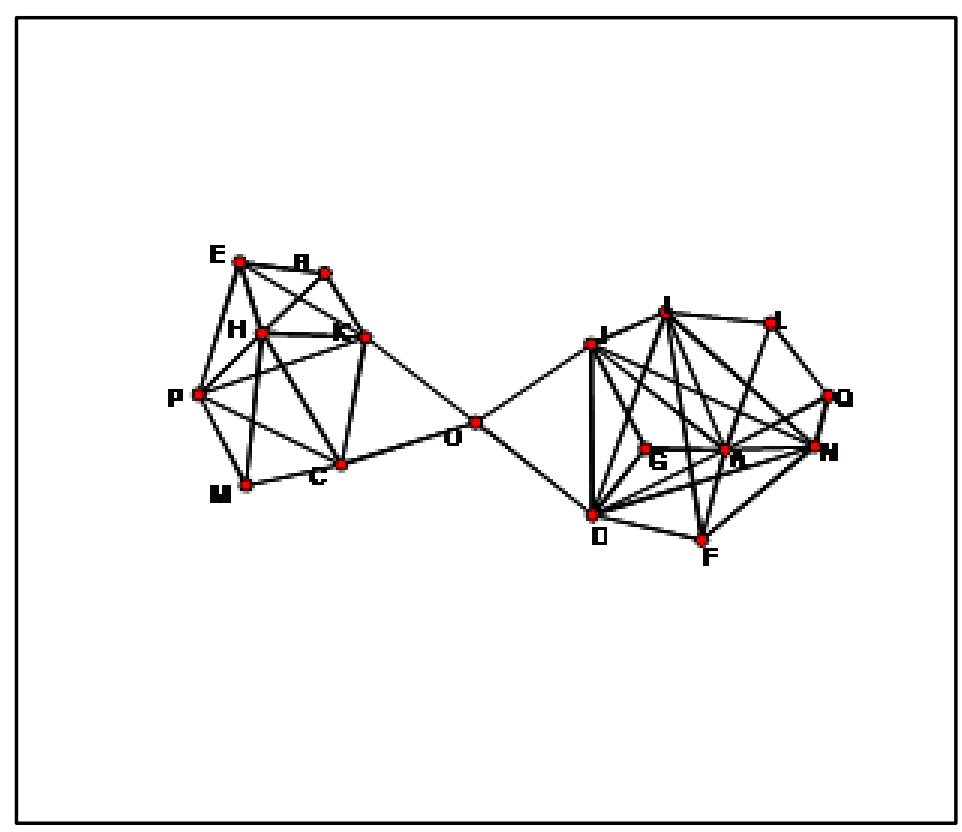

These two examples merely scratch the surface of the range of concepts used to characterize the shape of structures. Moreover, most groups do not take the clear prototypical shapes shown in Figures 3a and 3b, but rather are more complex and messy. A common research question is thus how close a network structure approximates a particular ideal type (e.g., Everett and Krackhardt, 2012), with different measures carrying distinct implications for the group.

In the special issue, the networks-as-a-whole approach is featured prominently in the article by Scott DeRue, Jennifer Nahrgang, and Susan Ashford (2015). This paper examines whether the network structure of group members’ perceptions of each other, as well as the psychological and structural conditions those perceptions create, influence the group’s emergent leadership structure (see Figure 2b), as well as individual leadership emergence. In a sample of MBA consulting teams followed over 7 weeks, they found that group topology (the network density and centralization of interpersonal perceptions of 
warmth and competence) affected the actions and reactions of individuals to each other, and served as the building blocks for the emergence of either dense or centralized leadership structures in groups. This study builds a conceptual and empirical bridge between literature on social psychological processes and group network structure, presenting a dynamic process by which leadership emerges as a function of how people perceive the group and are perceived by the group.

Figure 2b. An interdisciplinary perspective on leadership structure emergence: (DeRue, Nahrgang \& Ashford, 2015)

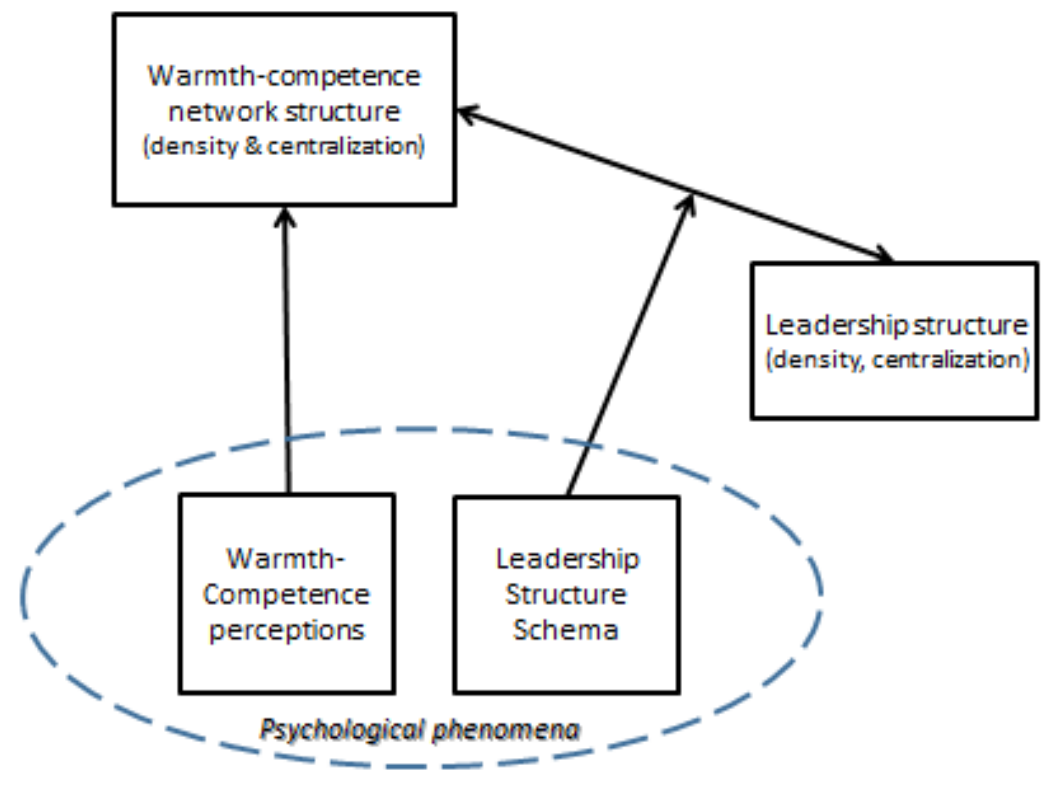

The Individuals in the Network (Positions-Roles). This second level of analysis is where we find a natural integration between network and psychological research, because this level speaks to individual differences, an important psychological area of inquiry. The individual-in-the-network level of analysis assesses the advantages or constraints one has because of one's position within the network. The network theories at this level are developed to explain what happens to the individual actor as a function of the network context in which they find themselves. However, each individual also brings a unique set of attributes such as age, education, experience, personal attitudes, beliefs, and personality with them to those social situations.With these psychological foundations, micro-level organizational behavior research 
is well positioned to ask questions such as, "Why do some people end up in a particularly advantageous network position?” and to be able to develop more nuanced answers than those currently provided by network theorists.

Two primary individual position constructs have dominated the theory building at this level of analysis: centrality (Freeman, 1979) and structural holes (Burt, 1992). Each provides unique insight into how people benefit from their position in a network, and each contributes to organizational studies in a manner distinct from a traditional focus on individual characteristics, theoretically and empirically. There are a variety of ways of conceiving of a person's network centrality (e.g., Borgatti and Everett, 2006). Degree centrality is the simplest: it is merely the count of the number of people in the network someone is directly tied to. Degree is used as an indicator of how active a person is in a network (Freeman, 1979; Brass, 1984; Opsahl, Agneessens, and Skvoretz, 2010). Because degree centrality does not require one to take into account the structural interdependencies in the overall network to assess it, one might conclude that it is not a true network measure. However, extensions of the concept add theoretical nuance; for example, eigenvector centrality is based on the idea that not all ties are created equal and that being tied to some people who are in turn tied to many others gives rise to more advantages than being tied to other less active network members (Bonacich, 1972). This extension became the foundation of Google’s “Page Rank” algorithm for finding sites on the network of internet hyperlinks that were likely to be important to the user's search (Brin and Page, 1998). Bonacich (1987) offered another important elaboration of this concept, power or beta centrality, to account for “negative networks," wherein those who were tied to powerful others are less powerful, not more powerful, because their more powerful neighbors could extract higher concessions from them during negotiations. This elaboration also accounts for the decaying effects of others' power as a function of how far away they are in the network. Thus, even the simplest network concept, degree centrality, can be expanded to take into account recursive effects of the importance of the actors one is tied to, directly and indirectly, providing elaborate structural approaches to measuring and theorizing power and status. 
While degree centrality and its extensions have been useful guides to understanding individual's advantages and contributions to group and organizational processes, betweenness centrality, and its oftused related measure, structural holes (Burt, 2005) has been most identified with individual success in a social network. Betweenness centrality captures the extent to which an individual lies on critical paths between others in the network. Returning to Figure 3b (the bow tie), we see that person A has the most ties (highest degree centrality). But we can also see that most person A’s contacts are also tied to each other. Thus, none of the people A is tied to are dependent on A to get a message to any of the others; they can easily go around A to reach their targets. Despite A’s popularity, we would say that A has low betweenness centrality in this network. Measures of structural holes and betweenness are conceptually linked and empirically correlated, but the research on structural holes has documented more directly that individuals surrounded by structural holes are more productive, develop more creative ideas, and tend to get promoted more quickly in organizations. There are, however, personal costs borne by occupying these positions; being the bridge between different groups (like $\mathrm{O}$ is in Figure 3b) can lead to role conflict and stress (e.g., Krackhardt, 1999).

The Dyads in the Network (Interpersonal Relationships). Extensive and compelling evidence that position within a network affects a person's opportunities and constraints has given rise to investigation of how a person ends up in a given position. Interesting questions include: How do dyadic network ties form, strengthen, weaken, or disappear? How do people take advantage of these principles and surround themselves with an effective network? Why do we choose particular others to be our friends or our work partners? The dyadic level of analysis treats the network structure itself as the outcome variable of interest. Several factors are invoked in answering these questions. First, the psychological principle of similarity-attraction (Berscheid, 1985), or homophily in the sociology literature (Hogue and Steinberg, 1995), refers to people’s preference for interacting with similar others. People of similar demographic characteristics (e.g., race, sex, age, education) and those who share similar beliefs, attitudes and personality (Williams and O'Reilly, 1998) tend to interact. Second, network tie formation and 
retention is influenced by propinquity, or physical distance between people (Allen, 1984). For example, two people in adjoining offices tend to communicate more often than two people with offices on separate floors. A third factor influencing interaction is affect or emotion. For instance, people tend to choose others to interact whom they like, even when doing so means avoiding people with superior competence and capability to help them accomplish their goals (Casciaro and Lobo, 2008). Answering dyadic-level questions of network formation and evolution will allow researchers to move from merely describing networks and their consequences to prescribing how to better manage and influence network structures, to create more adaptive topologies at the group level, or to achieve privileged positions at the individual level.

\section{Psychological phenomena}

To understand how the psychological perspective can be integrated usefully with the above network constructs, we focus now on four fundamental and most commonly studied psychological phenomena in organizational research: affect, cognition, individual differences (such as, personality and demographics), and motivation. These domains are overlapping and non-exhaustive, but they represent a large part of the psychological perspective on organizations. We describe each domain briefly before discussing how they may inform or be informed by network constructs.

Affect. Affect is an overarching term for experiences related to feeling, which include both affective states and traits (Barsade and Gibson, 2007). The term includes two types of affective states: emotions, which are short in duration and intense, and moods, which last longer, are more diffuse, and of which people are sometimes not aware of experiencing. Affect also includes affective traits; the most studied are individual differences in relatively stable tendencies to experience positive and negative emotions or moods, referred to as trait positive and negative affectivity (Watson, Clark and Tellegen, 1988). There are two basic approaches to studying affect. First, the affective circumplex model (Russell, 1980) a dimensional approach that emphasizes the interaction of two basic constructs -- emotional valence (positive or negative) and energy (high or low arousal), has generated considerable research over 
many years.. The other approach, currently gaining momentum, involves focusing on discrete emotions, such as anger, joy, companionate love, or envy (Lazarus and Cohen-Charash, 2001).

The role of affect in organizational behavior, a rapidly growing area of research, includes constructs such as emotional contagion, emotional intelligence, emotional labor, and emotional regulation (see Barsade and Gibson, 2007: 36-39, for a summary). Recent network research incorporates the psychology of affect into structural analyses of the emergence and consequences of dyadic task-related ties in organizational networks (e.g., Labianca and Brass, 2006; Casciaro and Lobo, 2008, 2014; Venkataramani, Labianca and Grosser, 2013; Gerbasi, Porath, Parker, Spreitzer, and Cross, forthcoming). However, the simple positive or negative and energizing or de-energizing affective reactions that network researchers have typically used to answer dyadic questions have not yet taken full advantage of the richness of psychological research on affect.

Cognition. Much organizational research has focused on cognitive processes, including decisionmaking, learning, memory, and perception. Cognition has been defined as the "capacity to mentally process, comprehend, and manipulate information—in short, the ability to learn.” (Reeve, 2007, p.77). Early cognitive research in organizations adopted a rational decision-making perspective that emphasized individual skills and the influence of group structure and process on group decisions. Notably, behavioral decision-theorists noted that people could and did deviate from rationality regularly, based upon biases and heuristics (Huff, 1995). Other approaches focused on the psychological construct of intelligence or general cognitive ability (“g”), as defined by Carroll (1993). Organizational behavior research into effects of the general construct of "g" on work performance found support for the idea that cognition helps explain performance at work (Hunter and Hunter, 1984; Schmidt and Hunter, 2004). In addition, research on learning in organizations at the individual (e.g., Argyris, 1982), group (e.g., Edmondson, 2002; Reagans, Argote, and Brooks, 2005; Gibson and Vermeulen, 2003) and organizational level (e.g., March, 1991) emphasizes the role of human cognition. 
A stream of psychological research referred to as social cognition is concerned with the perception, judgment, and memory of social stimuli; the effects of social and affective factors on information processing; and the behavioral and interpersonal consequences of cognitive processes. Social cognition research is particularly conducive to integration with network theory, in part due to the network concept of “cognitive social structures,” or CSS (Krackhardt, 1987). CSS representations of network phenomena in fact constitute a fourth level of analysis in network research. Represented as threedimensional cubes of network information provided by the N people in a network, wherein each person's perception of the entire network is represented as an NxN slice of that cube, CSS research claims that how people perceive their networks is a distinct and important variable. Beyond actual dyadic interactions in networks, CSS integrates structural and cognitive views of organizational behavior by considering the influence of people's perceptions of those networks. That is, while the network has structural effects on individuals, if a participant in the network believes the network to be different from what it actually is, that perception influences their perceived options and their behavior.

Network research on cognition has underscored that people’s behavior and attitudes change depending on their perceptions of the network (e.g., Kilduff \& Krackhardt, 1994). One of the articles in this special issue directly builds on this research tradition. Raina Brands, Jochen Menges, and Martin Kilduff (2015) began with findings from leadership schema research that indicated that subordinates confer leadership qualities on some, but not all, formally appointed leaders, based on the prototype they hold for leaders. These scholars were specifically interested in whether men and women may be differentially conferred with charismatic leadership qualities (Conger \& Kanungo, 1987). Brands et al. (2015) propose the concept of a leader-in-social-network-schema, and argue that it influences attributions of charismatic leadership by enabling individuals to match the gender of a leader and the structure of their network. In a series of experimental studies, they show that, when advice networks were depicted as centralized, female leaders were seen as less charismatic than male leaders, but when such networks were perceived to be cohesive, male leaders were seen as less charismatic than female leaders (see Figure 2c). By examining how perceptions of network structure influence perceivers' attributions, Brands et al. 
(2015) advance a cognitive social network approach to leadership, showing that "perceptions of agency and communality arise not only from men's and women's behavior but also from the social context within which the behaviors occur.” In sum, gender biases are influenced by network structure.

Figure 2c. An interdisciplinary perspective on charisma attributions (Brands, Menges, \& Kilduff, 2015)

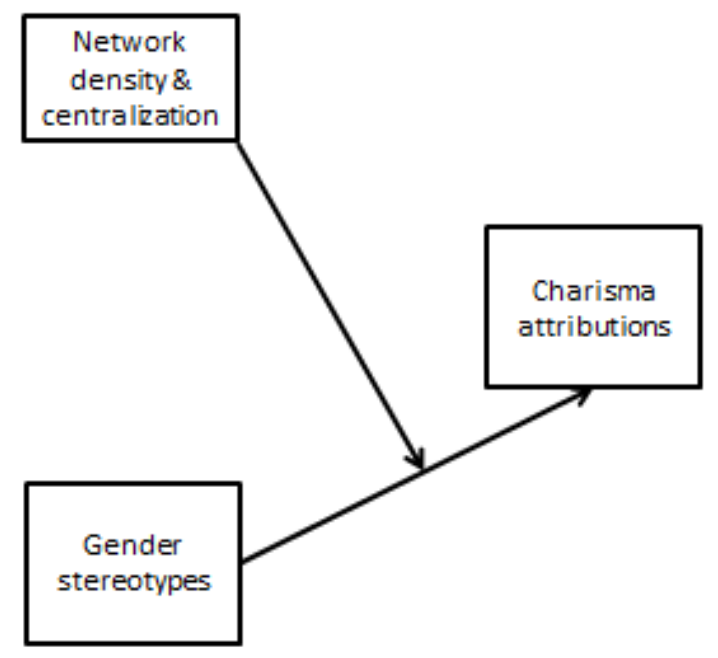

Network research has also explored predictors of the varieties of network perceptions, some of which lead to substantial misperceptions and inaccuracy. For example, there is a tendency to see more solid groupings and clusters of ties than actually exist (Kilduff et al., 2008). In recent years, research on network perception has seen a deeper consideration of how emotional and cognitive psychological processes inform our understanding of network perception. For example, Menon, Smith, and Thompson (2012) have shown that job threat can prime different recollections of one's social network contingent on a person's social status. High (low) status people faced with job threat recall large (small) networks with high (low) range, thus influencing their capacity to respond to the threat, which in turn can reinforce inequality.

Individual Differences. The psychological domain most integrated with network research is individual differences, particularly personality. Variables that capture personality and other individual differences present a natural fit with the interest in person versus situation that characterizes research on 
network structure -- and structuralism more generally. In particular, the personality trait of selfmonitoring -- the "active construction of public selves to achieve social ends" (Gangestad and Snyder, 2000, p. 546) to proactively behave in ways to fit the social situation -- has received much attention in network research (e.g., Mehra, Kilduff \& Brass, 2001; Sasovova, et al., 2010).

Adam Kleinbaum, Alexander Jordan and Pino Audia (2015) build on this tradition in the special issue by examining the established relationship between self-monitoring and an individual's brokerage position (e.g., Sasovova, et al. 2010), suggesting this relationship is influenced by others' perceptions of the focal individual. Based on the social value that others derive from feeling understood by an empathetic actor (Reis, Clark \& Holmes, 2004), Kleinbaum et al. (2015) reason that others’ perceptions of the extent to which the high self-monitor is also empathetic should strengthen the relationship with certain features of the ego's network (see Figure 2d). The rationale is that listening to others and a personal development focus are conducive to developing advantageous network positions. Based on online surveys of MBA students over seven weeks, the researchers found support for these ideas, demonstrating that self-monitoring was a strong predictor of brokerage position in a network for people perceived as empathetic; self-monitoring and empathy also predicted reciprocity of ties. In doing so, they refine the specification of social psychological models of network position. Extending the meta-analytic evidence they showed that brokers' ability to build a diverse set of ties rests in part on effectively conveying a sense of empathy to others, not only whether they are high self-monitors.. 
Figure 2d. A psychological perspective on network brokerage (Kleinbaum, Jordan \& Audia, 2015)

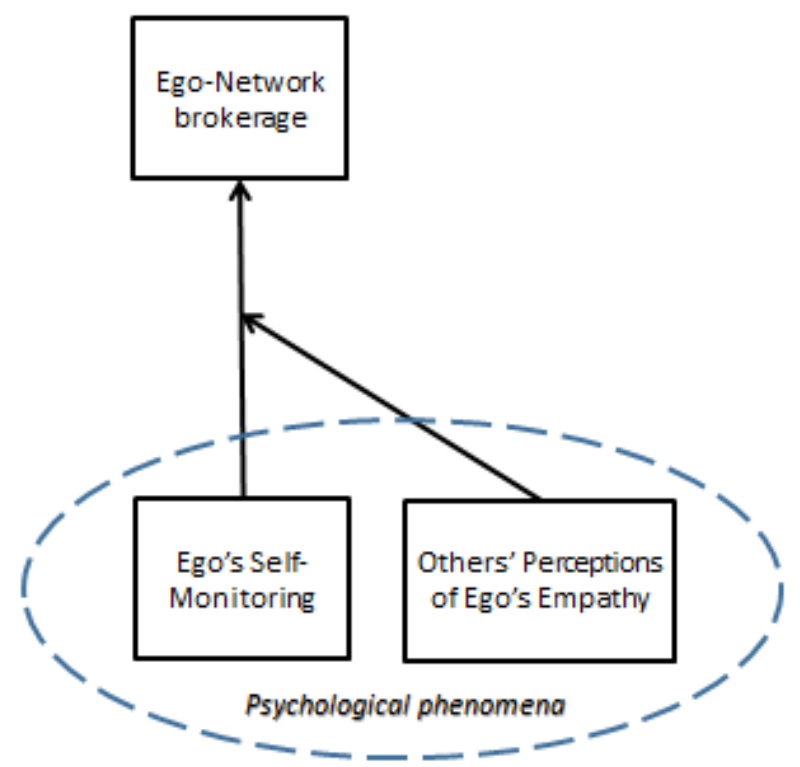

From a personality theory perspective, the "Big Five" is considered to be one of the most valid and stable overall summaries of personality (Costa \& McCrae, 1997; Goldberg,1981; Norman, 1963) and consists of the following five factors: "Extraversion or Surgency (talkative, assertive, energetic); Agreeableness (good-natured, cooperative, trustful); Conscientiousness (orderly, responsible, dependable); Emotional Stability versus Neuroticism (calm, not neurotic, not easily upset); Intellect or Openness (intellectual, imaginative, independent- minded)” (John, Srivastava, \& Pervin, 1999). Despite debate as to the best summary statements of personality (Costa \& McCrae, 1997) and recent research indicating that personality is more malleable in adulthood than was previously thought (Roberts, Donnellan \& Hill, 2013), trait personality theory, inclusive of the "Big Five” personality variables, has played a reliable and useful predictive role in organizational behavior (Barrick \& Mount, 1991). Other individual difference variables (including self-esteem, self-efficacy, and trait positive and negative affectivity as mentioned above), along with demographic variables (e.g., gender, race, and nationality) also fit usefully into this category. 
Figure 2e. Ameta-analysis of personality, network centrality, and performance (Fang, et al. 2015)

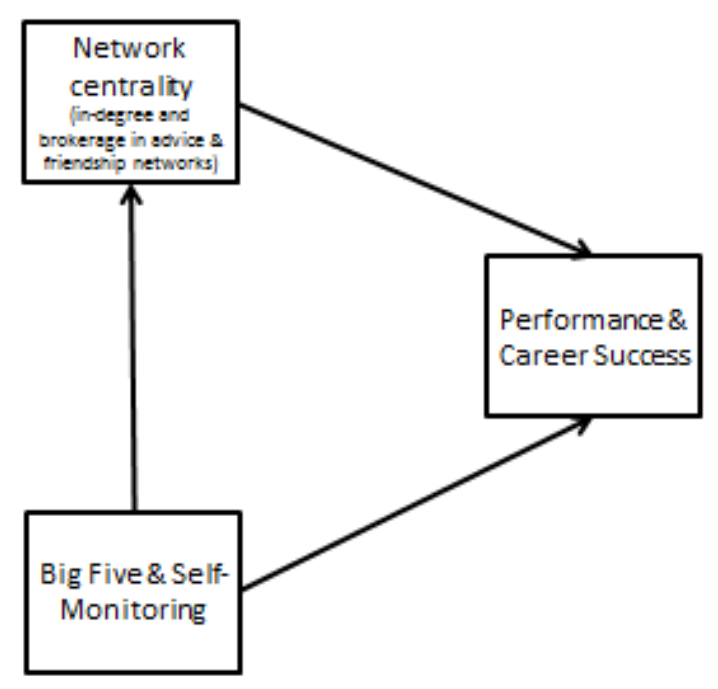

The special issue includes a meta-analysis by Fang and colleagues (2015) who examine whether the relationship between personality characteristics and work outcomes is mediated by network position, how personality affects network position, and how network position is related to work outcomes using 138 independent samples. The studies in the meta-analysis each include self-monitoring (Snyder, 1974) and the Big Five personality characteristics (extraversion, conscientiousness, openness to experience, agreeableness, and neuroticism, McCrae \& John, 1992). Work outcomes investigated included performance and career success, while network position is represented by in-degree centrality (the number of incoming ties an individual receives from others) and brokerage (i.e, structural holes). Relationships are investigated in both expressive (e.g., friendship) and instrumental (e.g., advice) networks. As depicted in Figure 2e, both personality and network centrality predict performance and career success directly, suggesting that both matter. But personality is only a modest predictor of indegree centrality and brokerage (explaining between 3 and 5 percent of the variance), and network position is only a partial mediator of the personality-outcome relationship.

Motivation. Psychological research includes myriad motivation theories. Kleinginna \& Kleinginna (1981), in their comprehensive review, outlined five major definitions of motivation: (1) 
phenomenological definitions that focus on the conscious, agentic processes involved in motivations (e.g. choosing to act); (2) physiological definitions that emphasize internal physical processes (e.g. neural processes, drives); (3) energizing definitions that emphasize arousal of energy that leads people to do things or continue to do things; (4) functional or directional definitions which emphasize goal-directed behavior, incentives and choice; and (5) vector definitions that emphasize the directionality that motivation takes, including how long it lasts (which also would incorporate temporal definitions).

Organizational behavior research, drawing from the above definitions, has conceptualized motivation as having three basic properties: (a) Directionality - the choice of which goals (of many) people decide to pursue (this includes studies of job choice, tasks employees focus upon, and absenteeism or voluntary turnover); (b) Intensity - the amount of energy or effort with which people pursue their goals (including task or work engagement); and (c) Persistence - how long people continue to work towards accomplishing their chosen goals (note that this incorporates the other two factors in that the choice to continue may encompass both direction and intensity) (Kanfer, 1990; 1995). Motivational theories within organizational theory also have been classified as content theories, which focus primarily on internal needs (physiological and psychological - e.g. Maslow, Herzberg, McClelland) that explain motivation, processes theories (behavioral reinforcement, equity and expectancy theories) that explain choice, effort and persistence, and a set of theories that help explain sustained motivation over time, including job design, goal-setting and incentive theories (Landy, 1985; Locke \& Latham, 2002).

Unlike affect, cognition, and personality, which, to varying degrees, have been integrated with the network perspective on organizations, the sophistication of psychological theory on motivation is still largely absent from network research, as this special issue also suggests. We elaborate on this gap below.

\section{Further avenues for network-psychological integration}

The articles in this special issue illustrate the types of insights gained through integrating psychological and network perspectives on organizational phenomena. Because of these articles, we now know more about which leaders will be perceived as charismatic in certain networks; when employees 
considering leaving their organization actually will turn over based on their connections with others; how social ties alter the relationship between personality and outcomes achieved at work; how perceptions influence the emergence of leadership structures in groups; and the role of empathy in connecting our individual characteristics to our network position.

The advances of the special issue notwithstanding, large swaths of network-psychological integration are not represented in this research forum, and are still largely unexplored in organizational research. These present potential avenues for further interdisciplinary exploration. We contemplate a small subset of such avenues here - notably providing examples of (1) theoretical models, (2) psychological phenomena, and (3) network phenomena that are rarely investigated in organizational research with an interdisciplinary perspective.

\section{Under-explored theoretical models}

The five articles in the special issue fall under one of two themes -- comprising a subset of the theoretical models represented in Figure 2. The first theme is a focus on how network characteristics alter relationships between psychological concepts, as shown in Brands, Menges and Kilduff (2015), Vardaman et al. (2015) and Fang et al., (2015). These articles clarify network-based boundary conditions for important relationships among cognition, attitudes, and personality on the one hand and behavior on the other. The second theme is a focus on relationships between a particular psychological variable and network characteristics, with psychological variables potentially changing the nature of that relationship. DeRue, Nahrgang and Ashford (2015) and Kleinbaum, Jordan and Audia (2015) illustrate this theme, contributing knowledge about the psychological processes that form building blocks of structures that emerge at the collective level.

Without exception, the articles in the special issue treat organizational phenomena--such as turnover, charisma attributions, or performance--as dependent variables. Yet, insight may be gained from reversing the causal direction to theorize the effect of organizational phenomena on network and psychological outcomes. For example, consider the effects of adhering to or deviating from the values of 
an organizational culture on an individual's network position in the organization, and his or her emotions and perceptions, as illustrated in Figure 2f. Consider cultural consensus analysis (Romney, Weller \& Batchelder, 1986), a network theory and method that defines and measures the extent to which certain beliefs, values and attitudes are shared by a group of individuals, and the extent to which any given individual in the group shares in the "consensus" or deviates from it. Although often used to define conditions under which greater agreement among individuals on right answers to a test indicates more knowledge on their part, cultural consensus analysis also can be used to study the level of shared cultural norms and values in an organization, and how close or distant an individual is from these shared values. This in turn raises intriguing questions. How does an individual's adherence or deviance from the organizational cultural consensus influence her capacity to be a bridge in organizational networks? How does it influence the emotions a person experiences? How do such emotion affect interpersonal relationships and resulting network position? Network-based concepts may shed light on how individual emotions, perceptions and behavior emerge in organizational life.

Figure 2f. Under-investigated theoretical links

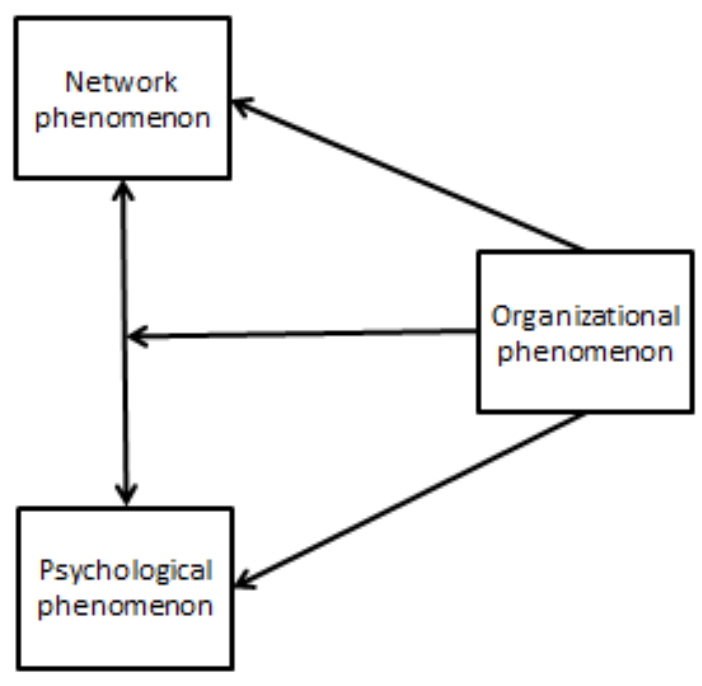

\section{Under-explored psychological phenomena}


Although the papers in the special issue use varied approaches and methods (survey-based field research, experiments, meta-analyses), their consistent inclusion of personality and interpersonal perceptions suggests that, among psychological constructs, these may be the most attractive for social network researchers. Many other submissions we received for the special issue similarly included personality constructs, underscoring the interest in these interactions. Personality attracts attention in network studies, despite finding low effect sizes in predicting network characteristics (Fang, et al., 2015).

In contrast with personality and cognition--and, to a lesser extent, affect--motivation is rarely addressed in network studies of organizations. When theorizing about the influence of strong ties, network research on knowledge transfer refers to (but does not measure) motivation (Granovetter, 1979). Affective closeness to someone is thought to motivate people to collaborate (Krackhardt, 1992) and to devote time and effort to transfer tacit and complex knowledge to that person (Hansen, 1999). Much remains to uncover about the role of motivation in networks, and how network characteristics may, in turn, affect motivation.

A psychological account of motivation can inform the long-standing sociological debate on the interplay of structural determinism and individual agency in social life, generally, and specifically in networks (Giddens, 1984; Bourdieu, 1991; Archer, 1995). For example, building on Kilduff and Tsai’s (2003) distinction between goal-directed and serendipitous networks, Casciaro, Gino and Kouchaki (2014) showed that, unlike social ties that emerge spontaneously, intentional networking in pursuit of professional goals can lower an individual's sense of moral purity, making him feel physically dirty, and discouraging forging social connections. These results imply that what motivates relational action can change the emotional experience of an individual and her resulting networks. Applied to network research on brokers, with its tendency to assume agency (Burt, 2005), these findings suggest that understanding the emergence and sustainability of brokerage behavior may require understanding whether brokers intentionally pursued the benefits of bridging structural holes or instead reacted to the demands and opportunities of operating at the boundaries of a social structure. 
Many similar questions remain open, and psychological theories of motivation and network research may invigorate one another in seeking to answer them. For instance, what is the role of envy as motivational impetus, and what network positions elicit envy? What network configurations operate as antecedents or consequences of pro-social and counterproductive behavior in organizations? What are the boundary conditions for such structural effects? How do regulatory focus versus goal-setting theory versus evolutionary theory shed light on agency in networks? Each of these questions could benefit from an interdisciplinary investigation.

\section{Under-explored network phenomena}

Although two of the five studies in the special issue considered the structural configuration of networks as a whole, this level of analysis has rarely been adopted in psychological studies of organizations. Most research linking psychological and network paradigms use an individual or dyadic level of analysis. One rare exception, which demonstrates the insights that we can derive from seeing psychological phenomena through a network perspective, is Schulte, Cohen and Klein’s (2012) study on the co-evolution of group psychological safety and team network structure. Much more can be done in this direction.

Thinking more about the interplay between network topology and psychology may expand our understanding of group affect. Group affect research shows that people gravitate towards affectivelysimilar others, because of factors including similarity attraction, affective convergence, emotional contagion and emotional culture (see Barsade \& Knight, 2015 for a review). Incorporating network theorizing may allow us to better specific group-affect configurations. Consider, for example, emotional culture-defined as the underlying assumptions, values and behavioral norms that reflect an array of discrete emotions in a group, and the appropriateness of expressing or suppressing these emotions in the social unit (Barsade \& O’Neill, 2014). Micro-organizational behavior scholars characterize emotional culture in terms of its mean or standard deviation in an organization. A network approach allows one to consider, theoretically and methodologically, more nuanced structural configurations that emotional 
culture can take, each with different substantive meaning and organizational implications. For example, an emotional culture structured like a relatively homogenous core-periphery network (as in Figure 3a) could have the same mean and standard deviation scores as an emotional culture structured as a bow-tie network (Figure 3b). But, unlike core-periphery structures, bow-tie emotional cultures might lead to an affective value clash (O’Reilly, 1989) of distinct emotional cultures: e.g., one high-joy/low-fear culture where employees are supposed to express positivity and enthusiasm; the other a low joy/high fear culture. What this example demonstrates is that, even in a field with fairly established findings, incorporating theorizing about networks as a whole allows greater precision in addressing countless questions. What are the shapes of these affective structures? How do differing affective structure shapes emerge? Are there dominant affective subgroups? Equal power groups? What are the consequences of these shapes? Do different shapes, even with the same density of affective ties, lead to more or less isolation or loneliness in the groups?

The network-as-a-whole approach may bring fresh theoretical insight to the study of other cognitive and cultural phenomena, and vice versa. Consider the topic of identity--a key mechanism in theory relating the external-to-internal tie (E-I) index with organizational crisis management (Krackhardt and Stern, 1988). In this work, organizations characterized by individuals with more external ties (ties across boundaries within organizations) relative to internal ties (ties connecting people within organizational units) will have more people who identify with the organization as a whole. In times of crisis, such an organization can weather the storm, because people -- identifying with the well-being of the organization as a whole -- engage in cooperative behavior firm wide.

While the E-I theory is sensible, it could be enriched by additional psychological constructs. For example, social psychological research shows that individuals affiliate with more than one identity group (Stryker and Burke, 2000). Ramarajan (2014), for example, argues that an “intrapersonal identity network” encompasses multiple identities considered simultaneously, and emphasizes the importance of relationships between identities. This conceptualization suggest that different sources of a person's identity operate as a system in which nodes (identities) are linked via ties (relationships) to form a whole 
(the identity network). Further, researchers have considered the behaviors or elements that comprise each identity (what are the elements of a professional identity? a racial identity?), as well as the relationships among these elements (Gibson, Dunlop, and Caprar, 2015). This more nuanced understanding recognizes that some elements of each identity may be connected and compatible, while others are compartmentalised or in conflict.

Combining this insight with the exactitude afforded by network constructs, we can envision an analysis of the topology of the elements of an intra-individual identity network. For example, a bow-tie configuration might mean that one's professional identity is only connected to one’s racial identity by a single "bridging element” that comprises both identities (e.g., perhaps social dominance?). We might then examine the E-I Index to assess the intrapersonal network of relationships among identity elements, to assess the proportion of ties bridging across identities compared to ties connecting elements within an identity. Perhaps, like groups with high E-I Index scores, individuals with high intra-psychic identity E-I scores (indicating a predominance of bridging ties) have greater ability to deal with or survive crises or conflicts. Such theorizing is only possible through a complex intermingling of psychological and network theory.

\section{Challenges of network-psychological interdisciplinary research}

The intention of our special issue was to bring together the study of social networks and psychological processes. This proved challenging. The two research programmes have developed into two distinct paradigms (defined as scientific communities, with groups of researchers similarly trained, sharing a worldview, including what is considered orthodoxy and thus impervious to challenge), making integration difficult (Kuhn, 1962). Conflicting paradigms are subject to dissensus over myriad issues, including appropriate topic choice, research design, and how to position one's hypothesizing within the research literature. All of these issues, and others, were evident in creating and managing the special issue. Our hope is that by surfacing these issues, we can offer a path toward a greater integration of the two research communities. 
Prior to posting a request for papers, our editorial team's members recognized these differences and resolved to bring together our disparate communities. One mechanism we employed was assigning each manuscript to co-handling editors, one primarily trained in the psychology of organizational behavior and the other in social networks, to ensure that our training and disciplinary background were not biasing our decisions and conclusions about particular manuscripts. In retrospect, this mechanism was slow, cumbersome, and largely unnecessary, because the two editors tended to agree on nearly every decision related to selecting publishable articles. Perhaps this points to a larger, shared aesthetic among organizational scholars who are interested in better understanding the intersection of these two domains. The co-editor structure proved most useful, however, in assigning appropriate reviewers. Because most editors rely on their communities to get high-quality reviewers, and because the editors were coming from disparate communities, the co-editor structure allowed each manuscript to get a wider variety of reviewers than it might otherwise receive. For many manuscripts, the network and psychologically trained reviewers agreed that the work was unpublishable within the timeframe of the special issue. However, for a few manuscripts, this broad set of reviews revealed the fissures between the communities to the editorial team, and we wish to offer some suggestions below at overcoming the these. Given our differences, how should we move forward with integrating our paradigms into meaningful scholarly advances? We provide suggestions in three arenas: topic choice, theoretical positioning, and research design.

\section{Topic Choice}

Kuhn (1962) points out that a crucial aspect of defining a paradigm is agreement on the puzzles that the community members will tackle. The differing research practices of the two scientific communities may however unwittingly preclude certain topics.

First, given the great difficulties of collecting field network data, policies (such as those recently espoused by American Psychological Association journals) that limit researchers' ability to use the same dataset for multiple studies restrict topic choice, and therefore interdisciplinary collaboration and progress. For example, a preferred method of tackling questions among network scholars is the use of 
prior datasets with which the community is familiar to ascertain whether a new theoretical mechanism explains an organizational phenomenon above and beyond earlier research. The Coleman et al. (1966) dataset has been used for this purpose many times. A strong norm for sharing datasets with other researchers exists among network researchers (e.g., the networks journal Connections publishes datasets for community use). If the re-use of datasets is not allowed in network-psychological studies of organizations, topic choice and collaboration will be restricted.

Second, scholars pursuing the integration of network and psychological perspectives on organizations may wish to reduce their focus on research questions at the individual level of analysis. A large proportion of the 93 submissions to the special were focused on individual differences. We encourage organizational scholars to extend the range of research topics they pursue to include higherlevel social structures, allowing for a deeper account of the complexity of the organizational context in which psychological phenomena are situated.

\section{Theoretical positioning}

How a study is grounded theoretically can also differ across the communities. Social network research tends to frame a study referring often to classics in organizational theory and the sociology of organizations, and drawing on logical reasoning, in addition to cited literature, to develop hypotheses. Micro-organizational scholars tend to develop predictions using a set of citations to support each component of a theoretical argument, with greater emphasis on more recent research. A compromise approach begins with recognizing that these two central tendencies exist (although exceptions certainly exist also), so as to draw from the strengths of each in an interdisciplinary manner. Historical grounding can sit alongside comprehensive recent literature, and moving from a broader theoretical frame to a more focused set of empirical studies is a means of satisfying both audiences.

\section{Research design}

Differences exist between our two scientific communities in how we approach research design that inhibit our ability to move forward together. We must either forge a compromise or embrace multi- 
method designs to meet the standards of inquiry each scientific community is most passionate about. An excellent way to do so is through collaborative research partnerships between scholars in micro organizational behavior and network traditions. This could help with defining proper statistical model specifications (particularly important to network researchers), specific measurement and testing of explanatory processes (particularly important to psychologically-trained scholars), and being open to understanding and testing hypotheses where they are most relevant--be that the field or the lab.

Defining proper model specification. Network research is concerned about data interdependence and finding special statistical models to treat the non-independence of network data. Some network reviewers feel that these are the only appropriate models to use (e.g., stochastic actor-oriented models). Yet, many of these models remain impractical for most researchers, requiring specialized training and constant updating. Psychological methods are also in flux, and debate about standards of evidence are ongoing. Tolerance and a spirit of learning are necessary on both sides if interdisciplinary work is to flourish.

On the importance of testing mediating processes. Organizational psychologists tend to be more likely to emphasize the direct measurement and testing of mechanisms to explain, for example, why sparse networks lead to being seen as having good ideas (cf., Burt, 2005). Network researchers, instead, tend to privilege deductive theorizing as the primary justification for proposed mechanisms, instead of relying on direct measures that may be arduous to collect in field settings. Both approaches to understanding mechanisms are valuable, and objections to them need to be reasonable, not driven by orthodoxy. An appreciation for both deductive reasoning, particularly when grounded in prior theoretical findings, and direct empirical testing is necessary to sustain interdisciplinary efforts.

Appropriate samples. Another area of disagreement has been what constitutes an "appropriate sample.” Network researchers typically prefer to collect data in the field, especially in organizations with working adults. Micro organizational behavior scholars are more open to using student and online 
samples (e.g., MTurk, Qualtrics Panel, e-Rewards). Future work might seek creative combinations of field and online data in multi-study projects.

\section{The way forward}

In this article we have underscored areas in which our scientific communities differ. However, although these differences are at times stark, we do not believe they are irreconcilable. One of the best ways to bring them together is through collaborative partnerships across disciplines. The theoretical and empirical standards of each research community are as stringent as they are different. For researchers rooted in one discipline to master the highly specific requirements of the other community will be exceptionally difficult. Meeting the expectations of both communities is more easily done when scholars from both disciplines join forces in collaborative research projects. Interestingly, almost all the submissions to this special issue were authored by teams of researchers who belonged to either the network or the psychological and micro-OB scientific community. This makes the interdisciplinary enterprise we wished to encourage much more arduous. Given the spirit of collaboration seen on our own editorial team, we know that it is possible to straddle the areas, but we need bonhomie and openness to interdisciplinary partnerships if we are to accomplish the ultimate goal of defining and executing exciting research at the confluence of networks and psychological processes. 


\section{REFERENCES}

Allen, T. J. (1984). Managing the flow of technology: Technology transfer and the dissemination of technological information within the R\&D organization. MIT Press Books.

Archer, M. S. (1995). Realist social theory: The morphogenetic approach. Cambridge University Press.

Argyris, C. (1982). Reasoning, learning, and action: Individual and organizational (pp. 85-101). San Francisco, CA: Jossey-Bass.

Barrick, M. R., \& Mount, M. K. (1991). The big five personality dimensions and job performance: a meta-analysis. Personnel psychology, 44(1), 1-26.

Barsade, S. G. \& Gibson, D. E. (2007). Why does affect matter in organizations? Academy of Management Perspectives, 21(1), 36-59.

Barsade, S. G. \& Knight, A. (2015). Group affect. Annual Review of Organizational Psychology and Organizational Behavior, 2:21-46.

Barsade, S. G \& O'Neill O. A. (2014). "What's Love Got to do with It?: The Influence of a Culture of Companionate Love in the Long-term Care Setting," Administrative Science Quarterly, 59, 551598.

Bavelas, A. (1950). Communication Patterns in Task Oriented Groups. Journal of the Acoustical Society of America 22 (6), 725-730.

Berscheid, E. (1985). Interpersonal attraction. Handbook of social psychology, 2, 413-484.

Bonacich, E. (1972). A theory of ethnic antagonism: The split labor market. American sociological Review, 547-559.

Bonacich, P. (1987). Power and centrality: A family of measures. American Journal of Sociology, 11701182.

Borgatti, S. P., \& Everett, M. G. (2006). A graph-theoretic perspective on centrality. Social networks, 28(4), 466-484.

Bourdieu, P. (1991). Language and symbolic power. Harvard University Press.

Brands, R. A., Menges, J. I., \& Kilduff, M. (2015). The Leader-in-Social-Network Schema: Perceptions of Network Structure Affect Gendered Attributions of Charisma. Organization Science, 26(4).

Brass, D.J. (1984). Being in the right place: A structural analysis of individual influence in an organization. Administrative Science Quarterly, 29, 518-539.

Brin, S., \& Page, L. (1998). The anatomy of a large-scale hypertextual Web search engine. Computer networks and ISDN systems, 30(1), 107-117.

Burt, R.S. (1992). Structural holes: The social structure of competition. Cambridge, MA: Harvard University Press. 
Burt, R.S. 2005. Brokerage and closure: An introduction to social capital. New York, NY: Oxford University Press.

Carroll, J. B. (1993). Human cognitive abilities: A survey of factor-analytic studies. Cambridge: Cambridge University Press.

Casciaro, T., Gino, F. \& Kouchaki, M. (2014). The contaminating effects of building instrumental ties: How networking can make us feel dirty. Administrative Science Quarterly, 59(4): 705-735.

Casciaro, T. \& Lobo, M.S. (2008). When competence is irrelevant: The role of interpersonal affect in task-related ties. Administrative Science Quarterly, 53(4), 655-684.

Casciaro, T. \& Lobo, M.S. (2014). Affective primacy in intraorganizational task networks. Organization Science, 26(2), 373-389.

Coleman, J. S., Katz. E. \& Menzel, H. (1966), Medical Innovation: A Diffusion Study, Inidianapolis, The Bobbs-Merril Company, Inc.

Conger, J.A., \& Kanungo R.N. (1987). Toward a behavioral theory of charismatic leadership in organizational settings. Academy of Management Review, 12(4), 637-647.

Costa, PT JR \& McCrae RR (1997). Longitudinal Stability in Adult Personality. In R. Hogan, J.A. Johnson \& S.R. Briggs (Eds.), Handbook of Personality Psychology, (pp. 269-290). Orlando, FL: Academic Press.

DeRue, D.S., Nahrgang, J.D., \& Ashford, S.J. (2015). Interpersonal perceptions and the emergence of leadership structures in groups: A network perspective. Organization Science, 26(4).

Edmondson, A.C. (2002). The local and variegated nature of learning in organizations: A group-level perspective. Organization Science, 13(2), 128-146.

Everett, M. G., \& Borgatti, S. P. (1999). The centrality of groups and classes.The Journal of mathematical sociology, 23(3), 181-201.

Everett, M. G., and Krackhardt, D. (2012). A second look at Krackhardt's graph theoretical dimensions on informal organizations. Social Networks, 34(2), 159-163.

Fang, R., Landis, B., Zhang, Z., Anderson, M. H., Shaw, J. D., \& Kilduff, M. (2015). Integrating Personality and Social Networks: A Meta-Analysis of Personality, Network Position, and Work Outcomes in Organizations. Organization Science.

Freeman, L. C. (1979). Centrality in social networks conceptual clarification.Social networks, 1(3), 215239.

Gangestad, S., \& Snyder, M. (2000) "Self-monitoring: Appraisal and reappraisal." Psychological Bulletin, 126, 530-555.

Gerbasi, A., Porath, C.L., Parker, A. Spreitzer, G., and Cross, Rob (forthcoming). Destructive DeEnergizing Relationships: How Thriving Buffers Their Effect on Performance. Journal of Applied Psychology. 
Gibson, C.B., \& Vermeulen, F. (2003). A healthy divide: Subgroups as a stimulus for team learning. Administrative Science Quarterly, 48, 202-239.

Giddens, A. (1984). The constitution of society: Outline of the theory of structuration. Univ of California Press.

Gioia, D.A., \& Pitre, E. (1990). Multiparadigm perspectives on theory building. Academy of Management Review, 15(4), 584-602.

Goldberg, L. R. (1981). Language and individual differences: The search for universals in personality lexicons. Review of personality and social psychology, 2(1), 141-165.

Granovetter, M. (1979). The theory-gap in social network analysis. Perspectives on Social Network Research, 501-518.

Griffeth, R.W., Hom, P.W., \& Gaertner, S. (2000). A meta-analysis of antecedents and correlates of employee turnover: Update, moderator tests, and research implications for the next millennium. Journal of Management, 26(3), 463-488.

Hansen, M. T. (1999). The search-transfer problem: The role of weak ties in sharing knowledge across organization subunits. Administrative Science Quarterly, 44(1), 82-111.

Harvey, O. J., White, B. J., Hood, W. R., \& Sherif, C. W. (1961). Intergroup conflict and cooperation: The Robbers Cave experiment (Vol. 10). Norman, OK: University Book Exchange.

Heider, F. (1958). The psychology of interpersonal relations. New York, NY: Wiley.

Hogue, A., \& Steinberg, L. (1995). Homophily of internalized distress in adolescent peer groups. Developmental Psychology, 31(6), 897-906.

Homans, G. C. (1961). Social behavior: Its elementary forms. Oxford, England: Harcourt, Brace and World.

Huff, A., S. (1995). Cognition in organizations. In The Blackwell Encyclopedic Dictionary of Organizational Behavior. (2nd ed., Vol VI., pp. 64-66). Malden, Massachusetts: Blackwell Publishers Inc.

Hunter, J. E., \& Hunter, R. F. (1984). Validity and utility of alternative predictors of job performance. Psychological Bulletin 96(1), 72-98.

John, O.P., Srivastava, S., \& Pervin, L.A. (1999). Handbook of personality: Theory and research. Guildford New York

Kanfer, R. (1990). Motivation theory and industrial and organizational psychology. In Dunnette, Marvin D. (Ed) and Hough, Leaetta M. (Eds), Handbook of industrial and organizational psychology, Vol. 1 (2nd ed.). , (pp. 75-170). Palo Alto, CA, US: Consulting Psychologists Press, xxvii, pp. 755.

Kanfer, R. (1995). Motivation. In The Blackwell Encyclopedic Dictionary of Organizational Behavior. (2nd ed., Vol VI., pp. 330-336). Malden, Massachusetts: Blackwell Publishers Inc. 
Kilduff, M., Crossland, C., Tsai, W., \& Krackhardt, D. 2008. Organizational network perceptions versus reality: A small world after all? Organizational Behavior and Human Decision Processes, 107, 15-28.

Kilduff, M., \& Krackhardt, D. (1994) Bringing the individual back in: A structural analysis of the internal market for reputation in organizations. Academy of Management Journal, 37(1), 87-108.

Kilduff, M. J., and W. Tsai, (2003) Social Networks and Organizations. London: Sage.

Kleinbaum, A.M., Jordan, A.H., Audia. P.G. (2015) An Altercentric Perspective on the Origins of Brokerage in Social Networks: How Perceived Empathy Moderates the Self-Monitoring Effect. Organization Science, 26(4).

Kleinginna Jr, P. R., \& Kleinginna, A. M. (1981). A categorized list of emotion definitions, with suggestions for a consensual definition. Motivation and emotion, 5(4), 345-379.

Krackhardt, D. (1987). Cognitive social structures. Social Networks, 9,109-134.

Krackhardt, D. (1992). The strength of strong ties: The importance of philos in organizations. Networks and Organizations: Structure, Form, and Action, 216-239.

Krackhardt, D. (1999) The ties that torture: Simmelian tie analysis in organizations. Research in the Sociology of Organizations, 16(1), 183-210.

Krackhardt, D. (2010). "Social Networks". In John M. Levine and Michael A. Hogg (eds.) Encyclopedia of Group Processes \& Intergroup Relations. Thousand Oaks, CA.: Sage, pp. 817-821.

Krackhardt, D. \& Stern, R. N. (1988) Informal networks and organizational crises: An experimental simulation. Social Psychology Quarterly, 51(2), 123-140.

Kuhn, T.S. The Structure of Scientific Revolutions. Chicago: University of Chicago Press, 1962.

Labianca, G., \& Brass, D.J. (2006). Exploring the social ledger: Negative relationships and negative asymmetry in social networks in organizations. Academy of Management Review, 31(3), 596-614.

Landy, F. J. (1985). Psychology of work behavior. Relations Industrielles / Industrial Relations, 40(4), 896-898.

Lazarus, R. S., \& Cohen-Charash, Y. (2001). Discrete emotions in organizational life. Emotions at work: Theory, research and applications for management, 45-81.

Leavitt, H. (1951). Some effects of certain communication patterns on group performance. Journal of Abnormal and Social Psychology, 46, 38-50.

Locke, EA; Latham, GP (2002). Building a practically useful theory of goal setting and task motivation A 35-year odyssey. American Psychologist, 57(9): 705-717

March, J.G. (1991). Exploration and exploitation in organizational learning. Organization Science, 2(1), Special Issue: Organizational Learning: Papers in Honor of (and by) James G. March, 71-87.

Mayhew, B. H. (1980). Structuralism versus individualism 1: Part 1, shadowboxing in the dark. Social Forces, 59(2), 335-375. 
McCrae, R.R., \& John, O. P. (1992). An introduction to the five-factor model and its applications. Journal of Personality, 60, 175-215.

Mehra, A., Kilduff, M., \& Brass, D. J. (2001). The social networks of high and low self-monitors: Implications for workplace performance. Administrative science quarterly, 46(1), 121-146.

Mitchell, T.R., Holtom, B.C., Lee, T.W., Sablynski, C.J., \& Erez, M. (2001). Why people stay: Using job embeddedness to predict voluntary turnover. Academy of Management Journal, 44(6): 11021121.

Moreno, J. L. (1961). The role concept, a bridge between psychiatry and sociology. American Journal of Psychiatry, 118(6), 518-523.

Norman, W. T. (1963). Toward an adequate taxonomy of personality attributes: Replicated factor structure in peer nomination personality ratings. The Journal of Abnormal and Social Psychology, 66(6), 574.

Opsahl, T., Agneessens, F., \& Skvoretz, J. (2010). Node centrality in weighted networks: Generalizing degree and shortest paths. Social Networks, 32(3), 245-251.

O'Reilly, C. (1989). Corporations, culture and commitment: Motivation and social control in organizations. California Management Review, 31, 9-25.

Ramarajan, L. (2014). "Past, Present and Future Research on Multiple Identities: Toward an Intrapersonal Network Approach." Academy of Management Annals, 8(1): 589-659.

Reagans, R., Argote, L., \& Brooks, D. (2005). Individual experience and experience working together: Predicting learning rates from knowing who knows what and knowing how to work together. Management Science, 51(6), 869-881.

Reeve, C. (2007). Cognitive abilities. In S. Rogelberg (Ed.), Encyclopedia of industrial and organizational psychology. (pp. 77-80). Thousand Oaks, CA: SAGE Publications, Inc.

Reis, H.T., Clark, M.S., \& Holmes, J.G. (2004). Perceived partner responsiveness as an organizing construct in the study of intimacy and closeness. In D.J. Mashek, A.P. Aron, (Eds.) Handbook of Closeness and Intimacy. (pp. 201-225). Mahwah, NJ: Lawrence Erlbaum Associates

Roberts, B.W., Donnellan, M.B., \& Hill, P.L. (2013). Personality trait development in adulthood. In Tennen, H., Suls, J., \& Weiner, I. B. (Eds.) Handbook of psychology, Vol. 5: Personality and social psychology (2nd ed.). (pp. 183-196). Hoboken, NJ, US: John Wiley \& Sons Inc.

Romney, A.K., Weller, S.C., \& Batchelder W.H. (1986). Culture as consensus: A theory of culture and informant accuracy. American Anthropologist, 88(2), 313-338.

Russell, J. A. (1980). A circumplex model of affect. Journal of personality and social psychology, 39(6), 1161-1178.

Sasovova, Z, Mehra, A., Borgatti, S.P., \& Schippers, M.C. (2010). Network churn: The effects of selfmonitoring personality on brokerage dynamics. Administrative Science Quarterly, 55(4): 639670. 
Schmidt, F.L. \& Hunter, J. (2004). General mental ability in the world of work: Occupational attainment and job performance. Journal of Personality and Social Psychology, 86(1), 162-173.

Schulte, M., Cohen, N.A., \& Klein, K.J. (2012). The coevolution of network ties and perceptions of psychological safety. Organization Science, 23 (2): 564-581.

Shaw, M. E. (1964). Communication networks. Advances in experimental social psychology, 1, 111-147.

Simmel, G. (1950). The sociology of Georg Simmel. New York, NY: Free Press.

Smith, E.B., Menon, T., \& L. Thompson. (2012). Status differences in the cognitive activation of social networks. Organization Science, 23(1): 67-82.

Snyder, M. (1974). The self-monitoring of expressive behavior. Journal of Personality and Social Psychology, 30, 526-537.

Stryker, S., \& Burke, P. J. (2000). The past, present, and future of an identity theory. Social Psychology Quarterly, 284-297.

Vardaman, J., Taylor, S., Allen, D., Gondo, M., \& Amis, J. (2015), Translating Intentions to Behavior: The Interaction of Network Structure and Behavioral Intentions in Understanding Employee Turnover. Organization Science, 26(4).

Venkataramani, V., Labianca, G., \& Grosser, T. (2013). Positive and negative workplace relationships, social satisfaction, and organizational attachment. Journal of Applied Psychology, 98(6), 10281039.

Watson, D., Clark, L. A., \& Tellegen, A. (1988). Development and validation of brief measures of positive and negative affect: The PANAS scales. Journal of Personality and Social Psychology, 54(6), 1063-1070.

Wellman, B., \& Berkowitz, S. D. (Eds.). (1988). Social structures: A network approach (Vol. 2). New York, NY: Cambridge University Press.

Williams, K., \& O'Reilly, C. A. (1998). The complexity of diversity: A review of 40 years of research. Research in Organizational Behavior, 21, 77-140. 